\title{
Fast solution of Cahn-Hilliard Variational Inequalities using Implicit Time Discretization and Finite Elements
}

\author{
Jessica Bosch ${ }^{\mathrm{a}, *}$, Martin Stoll ${ }^{\mathrm{a}, 1}$, Peter Benner ${ }^{\mathrm{a}, \mathrm{b}, 1}$ \\ ${ }^{a}$ Computational Methods in Systems and Control Theory, Max Planck Institute for \\ Dynamics of Complex Technical Systems, Sandtorstr. 1, 39106 Magdeburg, Germany \\ ${ }^{b}$ Mathematics in Industry and Technology, Chemnitz University of Technology, \\ Reichenhainer Str. 39/41, 09126 Chemnitz, Germany
}

\begin{abstract}
We consider the efficient solution of the Cahn-Hilliard variational inequality using an implicit time discretization, which is formulated as an optimal control problem with pointwise constraints on the control. By applying a semi-smooth Newton method combined with a Moreau-Yosida regularization technique for handling the control constraints we show superlinear convergence in function space. At the heart of this method lies the solution of large and sparse linear systems for which we propose the use of preconditioned Krylov subspace solvers using an effective Schur complement approximation. Numerical results illustrate the competitiveness of this approach.
\end{abstract}

Keywords: Cahn-Hilliard equation, double obstacle potential, PDE-constrained optimization, Moreau-Yosida regularization technique, semi-smooth Newton method, preconditioning 2000 MSC: 35R35, 49J15, 74S05, 35K55, 35K85, 90C33, 49N90, 80A22, $82 \mathrm{C} 26,65 \mathrm{M} 60,65 \mathrm{~F} 10,65 \mathrm{~N} 22,65 \mathrm{~F} 50$

\footnotetext{
*Tel.: +493916110 450; fax: +493916110453

Email addresses: bosch@mpi-magdeburg.mpg.de (Jessica Bosch), stollm@mpi-magdeburg.mpg.de (Martin Stoll), benner@mpi-magdeburg.mpg.de (Peter Benner)

${ }^{1}$ Tel.: +49 3916110 450; fax: +493916110453
} 


\section{Introduction}

The Cahn-Hilliard equation is a partial differential equation of fourth order which is used in materials science [38, 23], image processing [17] or chemistry [48]. It was originally introduced to model phase separation in binary alloys [30, 14] which occurs when the temperature of such a homogeneous mixture is rapidly quenched below a critical temperature. For time $t \in(0, T)$, with $T>0$ fixed, we consider a binary mixture of components $A$ and $B$ and define $u$ as the difference of the local concentrations between the two components. Hence $u$ satisfies $u \in[-1,1]$ with $u \equiv 1$ in the pure phase $A$ and $u \equiv-1$ in the pure phase $B$. We here study a diffuse phase transition, i.e. the region between the two phases has a certain width $b$, the so-called interface (phase field model). There is also the limit case $b \downarrow 0$ which gives the Mullins-Sekerka system (sharp interface model) [13, 23, 40]. Phase separation is modelled either by a smooth free energy, e.g. double-well potentials [18] such as

$$
\psi(u):=\frac{1}{4}\left(u^{2}-1\right)^{2},
$$

logarithmic potentials [15], or by a non-smooth double obstacle potential $[8,9]$

$$
\psi(u):=\left\{\begin{aligned}
\frac{1}{2}\left(1-u^{2}\right), & |u| \leq 1 \\
\infty, & |u|>1 .
\end{aligned}\right.
$$

Because of the disadvantage of the former that physically non-admissible values $|u|>1$ can be attained during the evolution we consider the latter (on which we can control the restriction $|u| \leq 1$ more precisely). Moreover, Oono and Puri found that in the case of deep quenches the double obstacle potential is better suited than the other potentials mentioned above, see [39]. After discretization in time, this leads to an optimal control problem with pointwise constraints on the control. In [31], Hintermüller, Hinze and Tber propose a function space-based algorithm which combines a MoreauYosida regularization technique for handling the control constraints with a semi-smooth Newton (SSN) method for solving the optimality systems of the resulting subproblems. This method allows for a convergence analysis in function space $[32,45]$, for which one expects a mesh-independent behaviour of the algorithm [33]. Hintermüller et al. use a semi-implicit time discretization, i.e. in particular an explicit treatment of the potential (1). In $[6,7,13]$ it is shown, that even though the time step can be arbitrarily large for semi-implicit systems, the results obtained for large time steps are highly 
inaccurate for capturing the evolution of the sharp interface model. Moreover in [5], Blank, Sarbu and Stoll relax the time step restriction arising from the implicit time discretization for the scalar Allen-Cahn variational inequalities with non-local constraints. This motivates us to use a fully implicit scheme for the discretization in time and the treatment of the potential (1).

As we will show in the course of this paper the solution of a linear system $A x=b$ with $A$ a real symmetric matrix is at the heart of this method. The sparse linear systems are usually of very large dimension and in combination with three-dimensional experiments the application of direct solvers such as UMFPACK [16] becomes infeasible. As a result iterative methods have to be employed (see e.g. [27, 42] for introductions to this field). We propose the use of a Krylov subspace solver. The convergence behaviour of the iterative scheme typically depends on the conditioning of the problem and the clustering of the eigenvalues. These properties can be enhanced using preconditioning techniques $P^{-1} A x=P^{-1} b$, where $P$ is an invertible matrix that is easy to invert and resembles $A$. In this paper, we provide an efficient preconditioner $P$ for the solution of Cahn-Hilliard variational inequalities using an effective Schur complement approximation and (algebraic) multigrid developed for elliptic systems $[20,42,41]$. The linear systems arising in the semi-smooth Newton method have the following saddle point block-structure

$$
A=\left[\begin{array}{cc}
-L & M \\
M & K
\end{array}\right]
$$

with $M \in \mathbb{R}^{N \times N}$ being symmetric and positive definite, $K \in \mathbb{R}^{N \times N}$ symmetric and positive semi-definite and $L \in \mathbb{R}^{N \times N}$ symmetric and possibly indefinite. Due to the possible indefiniteness of $L$, a non-symmetric Krylov subspace solver is our method of choice. For moderate sizes of $A$ and threedimensional problems direct solvers such as UMFPACK [16] can show outstanding performance.

The paper is organized as follows. In Section 2 we introduce the CahnHilliard variational inequality. In sections $3-6$ we follow the analysis presented in Hintermüller et al. [31], in which we emphasize the differences between the semi-implicit time-discrete problem therein and the implicit time-discrete problem which we explore. In detail, an implicit time-discrete problem is considered in Section 3. We show, that the time-discrete problem is equivalent to an optimal control problem whose regularized version is introduced and analysed in Section 4. In Section 5, we consider a semi-smooth Newton method to solve the regularized subproblems. We derive the linear 
systems arising from discretization using finite elements in Section 6. In Section 7 , we analyse the linear systems and propose preconditioning strategies for the saddle point problems. We additionally introduce preconditioners for the semi-implicit scheme derived in [31]. Section 8 illustrates the competitiveness of our preconditioners for both problem setups. In Section 9, we discuss alternative approaches and their properties in comparison to our method. Section 10 summarizes our findings.

\section{Cahn-Hilliard model}

The Cahn-Hilliard equation is derived as the $H^{-1}$-gradient flow of the Ginzburg-Landau energy

$$
E(u)=\int_{\Omega} \frac{\gamma \varepsilon}{2}|\nabla u|^{2}+\frac{1}{\varepsilon} \psi(u) \mathrm{d} x,
$$

where $\varepsilon>0$ is proportional to the thickness of the interfacial region and $\gamma>0$ is a constant related to the interfacial energy density. Using the obstacle potential $\psi$ in (1), which can be written via the indicator function as

$$
\psi(u)=\psi_{0}(u)+I_{[-1,1]}(u),
$$

where $\psi_{0}(u)=\frac{1}{2}\left(1-u^{2}\right)$, we get the following Cahn-Hilliard system

$$
\begin{aligned}
\partial_{t} u & =\Delta w \\
w & =-\gamma \varepsilon \Delta u+\frac{1}{\varepsilon}\left(\psi_{0}^{\prime}(u)+\mu\right) \\
\mu & \in \partial \beta_{[-1,1]}(u) \\
|u| & \leq 1 \\
\frac{\partial u}{\partial n}=\frac{\partial w}{\partial n} & =0 \quad \text { on } \partial \Omega,
\end{aligned}
$$

where $\partial \beta_{[-1,1]}(u)$ is the subdifferential of the non-smooth part $\beta_{[-1,1]}(u):=$ $\int_{\Omega} I_{[-1,1]}(u)$ of the energy $E$. The system $(2)-(6)$ can be formulated as a variational inequality

$$
\begin{aligned}
\left\langle\partial_{t} u, v\right\rangle+(\nabla w, \nabla v) & =0 \quad \forall v \in H^{1}(\Omega) \\
(w, v-u) & \leq \gamma \varepsilon(\nabla u, \nabla(v-u))+\frac{1}{\varepsilon}\left(\psi_{0}^{\prime}(u), v-u\right) \quad \forall v \in H^{1}(\Omega),|v| \leq 1
\end{aligned}
$$

$$
|u| \leq 1 \quad \text { a.e. in } \Omega
$$


for which existence, uniqueness and regularity of a solution was shown in [8]. Here $(\cdot, \cdot)$ and $\langle\cdot, \cdot\rangle$ stand for the $L^{2}(\Omega)$-inner product and the duality pairing of $H^{1}(\Omega)$ and $H^{1}(\Omega)^{*}$, respectively.

\section{Time-discrete Cahn-Hilliard system}

In contrast to Hintermüller et al. [31], we apply an implicit time discretization, i.e. we use the backward Euler discretization for the time derivative $\partial_{t} u$ and treat all the other terms in (7)-(9) implicitly. In particular, the potential $\psi$. Let $\tau>0$ denote the time step size and $t_{n-1}=(n-1) \tau, n \in \mathbb{N}$, discrete times, then for every time step we have to solve the time-discrete Cahn-Hilliard system

$$
\begin{aligned}
(u, v)+\tau(\nabla w, \nabla v) & =\left(u^{(n-1)}, v\right) \quad \forall v \in H^{1}(\Omega) \\
(w, v-u) & \leq \gamma \varepsilon(\nabla u, \nabla(v-u))+\frac{1}{\varepsilon}\left(\psi_{0}^{\prime}(u), v-u\right) \quad \forall v \in H^{1}(\Omega),|v| \leq 1
\end{aligned}
$$

$$
|u| \leq 1 \quad \text { a.e. in } \Omega \text {, }
$$

where we write $u^{(n)}=u$ and $w^{(n)}=w$.

Remark 1. The difference between the implicit and semi-implicit system is the discretization of the potential $\psi$. In the semi-implicit case we have

$$
\begin{aligned}
(u, v)+\tau(\nabla w, \nabla v) & =\left(u^{(n-1)}, v\right) \quad \forall v \in H^{1}(\Omega) \\
(w, v-u) & \leq \gamma \varepsilon(\nabla u, \nabla(v-u))+\frac{1}{\varepsilon}\left(\psi_{0}^{\prime}\left(u^{(n-1)}\right), v-u\right) \quad \forall v \in H^{1}(\Omega),|v| \leq 1 \\
|u| & \leq 1 \quad \text { a.e. in } \Omega .
\end{aligned}
$$

We now want to rewrite (10)-(12) as the first-order optimality system of an optimization problem. For this we define

$$
\begin{aligned}
\mathcal{K} & :=\left\{v \in H^{1}(\Omega):|v| \leq 1 \text { in } \Omega\right\} \\
\mathcal{V}_{0} & :=\left\{v \in H^{1}(\Omega):(v, 1)=0\right\} .
\end{aligned}
$$

Choosing $v=1$ in (10) we obtain the conservation of mass, i.e. $(u, 1)=$ $\left(u^{(n-1)}, 1\right)=m \quad \forall n \in \mathbb{N}$, which is a specific feature of the Cahn-Hilliard model. Without loss of generality, we assume that $m=0$ and $|\Omega|=1$ hold 
true. We further use the notation $\|\cdot\|$ for the $L^{2}$-norm. For (10)-(12) we obtain the following minimization problem

$$
\min _{(u, w) \in \mathcal{K} \times \mathcal{V}_{0}} J(u, w):=\frac{\gamma \varepsilon}{2}\|\nabla u\|^{2}+\frac{1}{\varepsilon} \int_{\Omega} \psi_{0}(u)+\frac{\tau}{2}\|\nabla w\|^{2} \quad \text { subject to }(10) .
$$

Let

$$
\mathcal{F}=\left\{(u, w) \in \mathcal{K} \times \mathcal{V}_{0}:(u, w) \text { achieves }(10)\right\}
$$

be the admissible set of $(\mathcal{P})$. Analogous to Lemma 3.1 in [31] we have the following result.

Lemma 1. The following properties hold true:

(i) $\mathcal{F} \neq \emptyset$ and $\mathcal{F} \subset \mathcal{V}_{0} \times \mathcal{V}_{0}$

(ii) $\mathcal{F}$ is a closed convex set of $H^{1}(\Omega) \times H^{1}(\Omega)$.

(iii) $J$ is strictly convex on $\mathcal{F}$, if $\tau<4 \gamma \varepsilon^{3}$.

(iv) For every sequence $\left(u_{m}, w_{m}\right)_{m \in \mathbb{N}}$ in $\mathcal{F}$ such that $\lim _{m \rightarrow \infty}\left\|u_{m}\right\|_{H^{1}}=\infty$ or $\lim _{m \rightarrow \infty}\left\|w_{m}\right\|_{H^{1}}=\infty$, we have $\lim _{m \rightarrow \infty} J\left(u_{m}, w_{m}\right)=\infty$, if $\tau<$ $4 \gamma \varepsilon^{3}$.

Proof. The proof for (i)-(ii) is the same as in [31].

(iii) Let $\left(u_{1}, w_{2}\right),\left(u_{2}, w_{2}\right) \in \mathcal{F}$ and $\alpha \in(0,1)$. Setting

$r(\alpha):=\alpha J\left(u_{1}, w_{1}\right)+(1-\alpha) J\left(u_{2}, w_{2}\right)-J\left(\alpha u_{1}+(1-\alpha) u_{2}, \alpha w_{1}+(1-\alpha) w_{2}\right)$,

we have

$$
r(\alpha)=\frac{\alpha(1-\alpha)}{2}\left(\gamma \varepsilon\left\|\nabla\left(u_{1}-u_{2}\right)\right\|^{2}+\tau\left\|\nabla\left(w_{1}-w_{2}\right)\right\|^{2}-\frac{1}{\varepsilon}\left\|u_{1}-u_{2}\right\|^{2}\right) .
$$

For $\left(u_{1}, w_{2}\right),\left(u_{2}, w_{2}\right) \in \mathcal{F}$ we choose $v=u_{1}-u_{2} \in H^{1}(\Omega)$ in $(10)$, subtract both equations from each other and get

$$
-\left\|u_{1}-u_{2}\right\|^{2}=\tau\left(\nabla\left(w_{1}-w_{2}\right), \nabla\left(u_{1}-u_{2}\right)\right) .
$$

Applying Young's inequality to (13), we obtain

$$
r(\alpha) \geq \frac{\alpha(1-\alpha)}{2}\left[\left(\gamma \varepsilon-\frac{\tau}{4 \beta \varepsilon}\right)\left\|\nabla\left(u_{1}-u_{2}\right)\right\|^{2}+\left(\tau-\frac{\tau \beta}{\varepsilon}\right)\left\|\nabla\left(w_{1}-w_{2}\right)\right\|^{2}\right]
$$


for all $\left(u_{1}, w_{2}\right),\left(u_{2}, w_{2}\right) \in \mathcal{F}$ and for all $\beta>0$. For the strict convexity we require $\tau<4 \gamma \varepsilon^{3}$ and choose $\beta$ such that $\gamma \varepsilon-\frac{\tau}{4 \beta \varepsilon}>0$ and $\tau-\frac{\tau \beta}{\varepsilon}>0$. Moreover, $r(\alpha)=0$ leads to $\left(u_{1}, w_{1}\right)=\left(u_{2}, w_{2}\right)$, see [31]. Consequently, $J$ is strictly convex on $\mathcal{F}$, provided that $\tau<4 \gamma \varepsilon^{3}$.

(iv) Let $(u, w) \in \mathcal{F}$. First of all, we have

$$
J(u, w)>\frac{\gamma \varepsilon}{2}\|\nabla u\|^{2}+\frac{\tau}{2}\|\nabla w\|^{2}-\frac{1}{2 \varepsilon}\|u\|^{2} .
$$

Choosing $v=u \in H^{1}(\Omega)$ in (10) and apply Young's as well as PoincaréFriedrichs inequality (note that $\mathcal{F} \subset \mathcal{V}_{0} \times \mathcal{V}_{0}$ ), we get

$J(u, w)>\left(\frac{\gamma \varepsilon}{2}-\frac{\tau}{8 \beta_{1} \varepsilon}-\frac{C_{p}}{8 \beta_{2} \varepsilon}\right)\|\nabla u\|^{2}+\left(\frac{\tau}{2}-\frac{\tau \beta_{1}}{2 \varepsilon}\right)\|\nabla w\|^{2}-\frac{\beta_{2}}{2 \varepsilon}\left\|u^{(n-1)}\right\|^{2}$

for all $(u, w) \in \mathcal{F}$ and for all $\beta_{1}, \beta_{2}>0$. Again we require $\tau<4 \gamma \varepsilon^{3}$ and choose $\beta_{1}, \beta_{2}$ such that $\frac{\gamma \varepsilon}{2}-\frac{\tau}{8 \beta_{1} \varepsilon}-\frac{C_{p}}{8 \beta_{2} \varepsilon}>0$ and $\frac{\tau}{2}-\frac{\tau \beta_{1}}{2 \varepsilon}>0$.

Remark 2. Note the necessity of the time step restriction $\tau<4 \gamma \varepsilon^{3}$ for the implicit scheme, which has not to be claimed for the semi-implicit one. Even though, the results obtained for large time steps with the semi-implicit system are highly inaccurate for capturing the evolution of the sharp interface model, see Section 8.2 or $[13,5,11]$.

The relation between $(\mathcal{P})$ and $(10)-(12)$ is established next.

Theorem 2. Let $\tau \in\left(0,4 \gamma \varepsilon^{3}\right)$. The problem $(\mathcal{P})$ has a unique solution $\left(u^{*}, w^{*}\right)$. Moreover, there exists a unique Lagrange multiplier $p^{*} \in H^{1}(\Omega)$, such that $w^{*}=p^{*}-\left(p^{*}, 1\right)$ and $\left(u^{*}, p^{*}\right)$ is a solution of (10)-(12). Conversely, if $\left(u^{*}, p^{*}\right)$ is a solution of (10)-(12), then $\left(u^{*}, w^{*}\right)$ with $w^{*}=p^{*}-\left(p^{*}, 1\right)$ is the unique solution of $(\mathcal{P})$.

Proof. The proof follows analogously to Theorem 3.2 in [31]. 


\section{Moreau-Yosida regularized problem}

Variational inequalities like (11) may be reformulated by introducing Lagrange multipliers associated with the constraints in $\mathcal{K}$. However, they are elements of $H^{1}(\Omega)^{*}$ and not allow a pointwise interpretation, which complicates the numerical treatment, see [5]. Motivated by [31], we replace the above optimization problem by its Moreau-Yosida regularized version

$$
\min _{(u, w) \in H^{1}(\Omega) \times \mathcal{V}_{0}} J_{c}(u, w) \quad \text { subject to }(10)
$$

with the objective

$$
J_{c}(u, w)=J(u, w)+\frac{1}{2 c}\|\max (0, u-1)\|^{2}+\frac{1}{2 c}\|\min (0, u+1)\|^{2},
$$

where $0<c \ll 1$ denotes the associated regularization/penalty parameter.

Theorem 3. Let $\tau \in\left(0,4 \gamma \varepsilon^{3}\right)$. The problem $\left(\mathcal{P}_{c}\right)$ has a unique solution $\left(u_{c}, w_{c}\right)$. Moreover, there exists a unique $p_{c} \in H^{1}(\Omega)$ such that

$$
\begin{array}{rlrl}
p_{c}-\left(p_{c}, 1\right) & =w_{c} & \\
\tau\left(\nabla p_{c}, \nabla v\right)+\left(u_{c}, v\right)-\left(u^{(n-1)}, v\right) & =0 & \forall v \in H^{1}(\Omega) \\
\gamma \varepsilon\left(\nabla u_{c}, \nabla v\right)+\left(\lambda_{c}\left(u_{c}\right), v\right)-\left(p_{c}, v\right)-\frac{1}{\varepsilon}(u, v) & =0 \quad \forall v \in H^{1}(\Omega),
\end{array}
$$

where $\lambda_{c}\left(u_{c}\right):=\lambda_{c}^{+}\left(u_{c}\right)+\lambda_{c}^{-}\left(u_{c}\right)$ with

$$
\lambda_{c}^{+}\left(u_{c}\right):=\frac{1}{c} \max \left(0, u_{c}-1\right) \quad \text { and } \quad \lambda_{c}^{-}\left(u_{c}\right):=\frac{1}{c} \min \left(0, u_{c}+1\right) .
$$

Conversely, if $\left(u_{c}, p_{c}\right)$ is a solution of (15)-(16), then $\left(u_{c}, w_{c}\right)$ with $w_{c}=$ $p_{c}-\left(p_{c}, 1\right)$ is the unique solution of $\left(\mathcal{P}_{c}\right)$.

Proof. By using the note in the proof of Theorem 4.1 in [31], that the functionals $u \rightarrow\|\max (0, u-1)\|^{2}$ and $u \rightarrow\|\min (0, u+1)\|^{2}$ are convex and Fréchet-differentiable on $H^{1}(\Omega)$, we can show that $\mathcal{F}_{c}$ and $J_{c}$ satisfy the analogue of Lemma 1 for $\left(\mathcal{P}_{c}\right)$. Hence, $\left(\mathcal{P}_{c}\right)$ has a unique solution $\left(u_{c}, w_{c}\right)$, provided that $\tau<4 \gamma \varepsilon^{3}$. The rest of the proof follows analogously to Theorem 4.1 in [31]. 
Proposition 4. Let $\left\{\left(u_{c}, w_{c}\right)\right\}_{c>0}$ be a sequence of solutions of $\left(\mathcal{P}_{c}\right)$ as $c \rightarrow 0$, where $\tau<4 \gamma \varepsilon^{3}$. Then there exists a subsequence still denoted by $\left\{\left(u_{c}, w_{c}\right)\right\}_{c>0}$ such that

$$
\left(u_{c}, w_{c}\right) \longrightarrow\left(u^{*}, w^{*}\right) \text { in } H^{1}(\Omega)
$$

as $c \rightarrow 0$, where $\left(u^{*}, w^{*}\right)$ is the unique solution of $(\mathcal{P})$. In particular, $u^{*}$ is the order parameter corresponding to the solution of (10)-(12).

Proof. Using the estimate for $J(u, w)$ in (iv) of Lemma 1, the proof follows analogously to Proposition 4.2 in [31].

\section{Semi-smooth Newton method}

We apply the function space-based algorithm motivated in [31] for solving the time-discrete Cahn-Hilliard problem. For a specified sequence $c \rightarrow 0$ we solve the optimality system (15)-(16), compactly written as

$$
F_{c}\left(u_{c}, w_{c}\right)=\left(F_{c}^{(1)}\left(u_{c}, w_{c}\right), F_{c}^{(2)}\left(u_{c}, w_{c}\right)\right)=0
$$

for every $c$ by a semi-smooth Newton algorithm. In (17), the components are defined by

$$
\begin{aligned}
& \left\langle F_{c}^{(1)}(u, w), v\right\rangle=\tau(\nabla w, \nabla v)+(u, v)-\left(u^{(n-1)}, v\right) \\
& \left\langle F_{c}^{(2)}(u, w), v\right\rangle=\gamma \varepsilon(\nabla u, \nabla v)+\left(\lambda_{c}(u), v\right)-(w, v)-\frac{1}{\varepsilon}(u, v)
\end{aligned}
$$

for all $u, w, v \in H^{1}(\Omega)$. Due to the presence of the max- and min-operators in the definition of $\lambda_{c}, F_{c}$ is not Fréchet-differentiable. However, it satisfies the weaker notion of Newton differentiability, see $[32,31]$.

Definition 1 (Definition 5.1 in [31]). Let $X$ and $Z$ be Banach spaces, $D \subset X$ an open subset. A mapping $F: D \rightarrow Z$ is called Newton-differentiable in $U \subset D$ if there exists a family of mappings $G: U \rightarrow Z$ such that

$$
\lim _{h \rightarrow 0} \frac{\|F(x+h)-F(x)-G(x+h) h\|_{Z}}{\|h\|_{X}}=0 \quad \forall x \in U .
$$

The operator $G$ is called a Newton derivative of $F$ on $U$. 
For such mappings, the following convergence result for the (semi-smooth) Newton iteration

$$
x^{(k+1)}=x^{(k)}-G\left(x^{(k)}\right)^{-1} F\left(x^{(k)}\right), \quad k=0,1, \ldots
$$

holds true, where $G$ is a Newton derivative of $F$. For its proof we refer to Theorem 1.1 in [32].

Theorem 5. Let $x^{*}$ be a solution of $F(x)=0$ and suppose that $F: D \subset X \rightarrow$ $Z$ is Newton-differentiable in a neighbourhood $U$ of $x^{*}$ with $\left\{\left\|G(x)^{-1}\right\|_{\mathcal{L}(Z, X)}\right.$ : $x \in U\}$ bounded. Then the sequence $\left\{x^{(k)}\right\}_{k \in \mathbb{N}}$ generated by (18) converges superlinearly to $x^{*}$ provided that $\left\|x^{(0)}-x^{*}\right\|_{X}$ is sufficiently small.

Lemma 6. The mapping $F_{c}: H^{1}(\Omega) \times H^{1}(\Omega) \rightarrow H^{1}(\Omega)^{*} \times H^{1}(\Omega)^{*}$ is Newtondifferentiable. Furthermore, the operator $G_{c}(u, w)$ given by

$$
\left\langle G_{c}(u, w)(\delta u, \delta w),(\phi, \psi)\right\rangle=\left(\begin{array}{c}
\tau(\nabla \delta w, \nabla \phi)+(\delta u, \phi) \\
\gamma \varepsilon(\nabla \delta u, \nabla \psi)+\frac{1}{c}\left(\chi_{\mathcal{A}(u)} \delta u, \psi\right)-(\delta w, \psi)-\frac{1}{\varepsilon}(\delta u, \psi)
\end{array}\right)
$$

serves as a Newton-derivative for $F_{c}$, where $\chi_{\mathcal{A}(u)}$ is the characteristic function of the set

$$
\mathcal{A}(u):=\{x \in \Omega:|u(x)|>1\} .
$$

For the proof we refer to Lemma 5.3 in [31] and Proposition 4.1 in [32].

Lemma 7. Let $\tau<4 \gamma \varepsilon^{3}$. For given $u \in H^{1}(\Omega)$ and $\left(y_{1}, y_{2}\right) \in H^{1}(\Omega)^{*} \times$ $H^{1}(\Omega)^{*}$, the optimization problem

$$
\begin{array}{rl}
\min _{(\delta u, \delta p) \in H^{1}(\Omega) \times \mathcal{V}_{0}} & J(\delta u, \delta p)+\frac{1}{c}\left(\chi_{\mathcal{A}(u)} \delta u, \delta u\right)-\left\langle y_{2}, \delta u\right\rangle \\
\text { subject to } & \tau(\nabla \delta p, \nabla \phi)+(\delta u, \phi)=\left\langle y_{1}, \phi\right\rangle \quad \forall \phi \in H^{1}(\Omega)
\end{array}
$$

admits a unique solution $(\delta u, \delta p)$. Moreover, there exists a unique $\delta w \in$ $H^{1}(\Omega)$ such that

$$
\begin{aligned}
\tau(\nabla \delta w, \nabla \phi)+(\delta u, \phi) & =\left\langle y_{1}, \phi\right\rangle \\
\gamma \varepsilon(\nabla \delta u, \nabla \psi)+\frac{1}{c}\left(\chi_{\mathcal{A}(u)} \delta u, \psi\right)-(\delta w, \psi)-\frac{1}{\varepsilon}(\delta u, \psi) & =\left\langle y_{2}, \psi\right\rangle
\end{aligned}
$$

for all $\phi, \psi \in H^{1}(\Omega)$. Conversely, if $(\delta u, \delta w)$ is a solution of (19)-(20), then $(\delta u, \delta p)$ with $\delta p=\delta w-(\delta w, 1)$ is the unique solution of $\left(\mathcal{P}_{G_{c}}\right)$. 
Proof. One proceeds as in the proofs of Theorems 2 and 3.

Lemma 8. The semi-smooth Newton method (18) (with $F$ and $G$ replaced by $F_{c}$ and $\left.G_{c}\right)$ converges superlinearly to $\left(u_{c}, w_{c}\right)$, the solution of $(17)$, provided that $\left\|\left(u^{(0)}, w^{(0)}\right)-\left(u_{c}, w_{c}\right)\right\|_{H^{1}(\Omega) \times H^{1}(\Omega)}$ is sufficiently small and $\tau<4 \gamma \varepsilon^{3}$.

Proof. From Lemma 7 we deduce that for all $(u, w) \in H^{1}(\Omega) \times H^{1}(\Omega)$, $G_{c}(u, w)$ is invertible, i.e. for given $\left(y_{1}, y_{2}\right) \in H^{1}(\Omega)^{*} \times H^{1}(\Omega)^{*}$, there exists a unique pair $(\delta u, \delta w) \in H^{1}(\Omega) \times H^{1}(\Omega)$, such that (19)-(20) is satisfied. Taking $(\phi, \psi)=(\delta w, \delta u)$ in $(19)-(20)$ and adding the two equations, we obtain

$$
\begin{aligned}
\gamma \varepsilon\|\nabla \delta u\|^{2}+\tau\|\nabla \delta w\|^{2} & =\left\langle y_{1}, \delta w\right\rangle+\left\langle y_{2}, \delta u\right\rangle-\frac{1}{c} \underbrace{\left(\chi_{\mathcal{A}(u)} \delta u, \delta u\right)}_{\geq 0}+\frac{1}{\varepsilon}\|\delta u\|^{2} \\
& \leq\left\langle y_{1}, \delta w\right\rangle+\left\langle y_{2}, \delta u\right\rangle+\frac{1}{\varepsilon}\|\delta u\|^{2} .
\end{aligned}
$$

Choosing $\phi=\delta u$ in (19) and applying Young's inequality with $\beta_{1} \in\left(\frac{\tau}{4 \gamma \varepsilon^{2}}, \varepsilon\right)$ (note that $\tau<4 \gamma \varepsilon^{3}$ ), we get

$$
\begin{aligned}
& \underbrace{\left(\gamma \varepsilon-\frac{\tau}{4 \beta_{1} \varepsilon}\right)}_{\geq 0}\|\nabla \delta u\|^{2}+\underbrace{\left(\tau-\frac{\tau \beta_{1}}{\varepsilon}\right)}_{\geq 0}\|\nabla \delta w\|^{2} \\
\leq & \left\langle y_{1}, \delta w\right\rangle+\left\langle y_{2}, \delta u\right\rangle+\frac{1}{\varepsilon}\left\langle y_{1}, \delta u\right\rangle \\
\leq & \frac{1}{4 \beta_{2}}\left(\|\delta w\|^{2}+\|\nabla \delta w\|^{2}\right)+\frac{1}{4 \beta_{3}}\left(\|\delta u\|^{2}+\|\nabla \delta u\|^{2}\right)+C\left(\left\|y_{1}\right\|_{H^{1}(\Omega)^{*}}^{2}+\left\|y_{2}\right\|_{H^{1}(\Omega)^{*}}^{2}\right)
\end{aligned}
$$

where we used Cauchy-Schwarz's and Young's inequality for the last result and the constant $C>0$ possibly depends on $\varepsilon, \gamma, \tau, c, \beta_{1}, \beta_{2}$ or $\beta_{3}$, but not on $\delta u$ or $\delta w$. Taking $(\phi, \psi)=(1,1)$ in $(19)-(20)$, we get

$$
\begin{aligned}
(\delta u, 1) & =\left\langle y_{1}, 1\right\rangle \\
(\delta w, 1) & =\frac{1}{c}\left(\chi_{\mathcal{A}(u)} \delta u, 1\right)-\frac{1}{\varepsilon}\left\langle y_{1}, 1\right\rangle-\left\langle y_{2}, 1\right\rangle .
\end{aligned}
$$

From (22)-(23) and (21), Poincaré-Friedrichs and again Young's inequality, it follows that

$$
\|(\delta u, \delta w)\|_{H^{1}(\Omega) \times H^{1}(\Omega)} \leq C\left(\left\|y_{1}\right\|_{H^{1}(\Omega)^{*}}+\left\|y_{2}\right\|_{H^{1}(\Omega)^{*}}\right) .
$$


For $\max \left(\left\|y_{1}\right\|_{H^{1}(\Omega)^{*}},\left\|y_{2}\right\|_{H^{1}(\Omega)^{*}}\right) \leq \beta$ for some constant $\beta>0$, we consequently have

$$
\left\|G_{c}^{-1}(u, w)\right\|_{\mathcal{L}\left(\left(H^{1}(\Omega)^{*}\right)^{2},\left(H^{1}(\Omega)\right)^{2}\right)} \leq \hat{C} \quad \forall(u, w) \in H^{1}(\Omega) \times H^{1}(\Omega)
$$

with some constant $\hat{C}>0$ possibly depending on $\varepsilon, \gamma, \tau, c, \beta_{1}, \beta_{2}, \beta_{3}$ or $\beta$, but not on $u$ or $w$. Thus $F_{c}$ with associated Newton derivative $G_{c}$ fulfills the conditions of Theorem 5, which completes the proof.

We now want to discretize the optimization problem and then discuss its efficient solution.

\section{Finite-element approximation}

We discretize (15)-(16) by finite elements [44]. In the following we assume for simplicity that $\Omega$ is a polyhedral domain. Generalizations to curved domains are possible using boundary finite elements with curved faces. Let $\left\{\mathcal{R}_{h}\right\}_{h>0}$ be a triangulation of $\Omega$ into disjoint open rectangular elements. The use of rectangles is established by the fact, that we perform the implementation with deal.II [1]. Furthermore, we define $\mathcal{R}_{h}$ to have maximal element size $h:=\max _{R \in \mathcal{R}_{h}}\{\operatorname{diam}(R)\}$ and we set $J_{h}$ to be the set of nodes of $\mathcal{R}_{h}$ and $p_{j} \in J_{h}$ to be the coordinates of these nodes. We approximate the infinite dimensional space $H^{1}(\Omega)$ by the finite dimensional space

$$
S_{h}:=\left\{\phi \in C^{0}(\bar{\Omega}):\left.\phi\right|_{R} \in Q_{1}(R) \quad \forall R \in \mathcal{R}_{h}\right\} \subset H^{1}(\Omega),
$$

of continuous, piecewise linear functions, where e.g. for dimension $d=2$ we have $Q_{1}=\operatorname{span}\left\{x^{\alpha_{i}} y^{\alpha_{i}}: \alpha_{i} \in\{0,1\}, i=1,2\right\}$. To each $p_{j} \in J_{h}$ we associate the nodal basis function $\varphi_{j} \in S_{h}$ with the property $\varphi_{j}\left(p_{i}\right)=\delta_{i j}, i, j=$ $1, \ldots, N$. The discretized version of the penalized problem (15)-(16) consists in finding $\left(u_{c, h}, w_{c, h}\right) \in S_{h} \times S_{h}$ such that

$$
\begin{array}{ll}
\left\langle F_{c, h}^{(1)}\left(u_{c, h}, w_{c, h}\right), v_{h}\right\rangle=0 & \forall v_{h} \in S_{h} \\
\left\langle F_{c, h}^{(2)}\left(u_{c, h}, w_{c, h}\right), v_{h}\right\rangle=0 & \forall v_{h} \in S_{h},
\end{array}
$$

where the components are

$$
\begin{aligned}
& \left\langle F_{c, h}^{(1)}\left(u_{c, h}, w_{c, h}\right), v_{h}\right\rangle=\tau\left(\nabla w_{c, h}, \nabla v_{h}\right)+\left(u_{c, h}, v_{h}\right)_{h}-\left(u_{h}^{(n-1)}, v_{h}\right)_{h} \\
& \left\langle F_{c, h}^{(2)}\left(u_{c, h}, w_{c, h}\right), v_{h}\right\rangle=\gamma \varepsilon\left(\nabla u_{c, h}, \nabla v_{h}\right)+\left(\lambda_{c}\left(u_{c, h}\right), v_{h}\right)_{h}-\left(w_{c, h}, v_{h}\right)_{h}-\frac{1}{\varepsilon}\left(u_{c, h}, v_{h}\right)_{h} .
\end{aligned}
$$


The semi-inner product $(\cdot, \cdot)_{h}$ on $C_{0}(\bar{\Omega})$ is defined by

$$
(f, g)_{h}:=\int_{\Omega} \pi_{h}(f(x) g(x)) \mathrm{d} x=\sum_{i=1}^{N}\left(1, \varphi_{i}\right) f\left(p_{i}\right) g\left(p_{i}\right) \quad \forall f, g \in C_{0}(\bar{\Omega}),
$$

where $\pi_{h}: C_{0}(\bar{\Omega}) \rightarrow S_{h}$ is the Lagrange interpolation operator. Within our finite-element framework, for a given $\left(u_{h}, w_{h}\right) \in S_{h} \times S_{h}$, every step of the semi-smooth Newton method for solving (24)-(25) requires to compute $\left(\delta u_{h}, \delta w_{h}\right) \in S_{h} \times S_{h}$ satisfying

$$
\begin{aligned}
\tau\left(\nabla \delta w_{h}, \nabla v_{h}\right)+\left(\delta u_{h}, v_{h}\right)_{h} & =-F_{c, h}^{(1)}\left(u_{h}, w_{h}\right) \\
\gamma \varepsilon\left(\nabla \delta u_{h}, \nabla v_{h}\right)+\frac{1}{c}\left(\chi_{\mathcal{A}\left(u_{h}\right)}^{h} \delta u_{h}, v_{h}\right)_{h}-\left(\delta w_{h}, v_{h}\right)_{h}-\frac{1}{\varepsilon}\left(\delta u_{h}, v_{h}\right)_{h} & =-F_{c, h}^{(2)}\left(u_{h}, w_{h}\right)
\end{aligned}
$$

for all $v_{h} \in S_{h}$, where $\chi_{\mathcal{A}\left(u_{h}\right)}^{h}:=\sum_{i=1}^{N} \chi_{\mathcal{A}\left(u_{h}\right)}^{h}\left(p_{i}\right) \varphi_{i}$ with $\chi_{\mathcal{A}\left(u_{h}\right)}^{h}\left(p_{i}\right)=0$ if $-1 \leq u_{h}\left(p_{i}\right) \leq 1$ and $\chi_{\mathcal{A}\left(u_{h}\right)}^{h}\left(p_{i}\right)=1$ otherwise. In matrix form, the linear system $(26)-(27)$ reads

$$
\begin{gathered}
{\left[\begin{array}{cc}
-\gamma \varepsilon K-\frac{1}{c} G_{A} M G_{A}+\frac{1}{\varepsilon} M & M \\
M & \tau K
\end{array}\right]\left[\begin{array}{c}
u^{(k+1)} \\
w^{(k+1)}
\end{array}\right]} \\
=\left[\begin{array}{c}
-\frac{1}{c}\left(G_{A^{+}} M G_{A^{+}} e-G_{A^{-}} M G_{A^{-}} e\right) \\
M u^{\text {old }}
\end{array}\right],
\end{gathered}
$$

where $e=(1, \ldots, 1) \in \mathbb{R}^{N}, u^{(k+1)}, w^{(k+1)} \in \mathbb{R}^{N}$ and $u^{\text {old }} \in \mathbb{R}^{N}$ is the solution from the previous time step. The lumped mass matrix and the stiffness matrix are defined as

$$
M_{i, j}:=\left(\varphi_{i}, \varphi_{j}\right)_{h} \quad \text { and } \quad K_{i, j}:=\left(\nabla \varphi_{i}, \nabla \varphi_{j}\right) \quad \forall i, j=1, \ldots, N,
$$

respectively, and

$$
\begin{gathered}
G_{A}=G_{A}\left(u^{(k)}\right)=\operatorname{diag}\left(\begin{array}{ll}
1, & \text { if }\left|u^{(k)}\left(p_{i}\right)\right|>1 \\
0, & \text { otherwise }
\end{array}\right) \\
G_{A^{+}}=G_{A^{+}}\left(u^{(k)}\right)=\operatorname{diag}\left(\begin{array}{ll}
1, & \text { if } u^{(k)}\left(p_{i}\right)>1 \\
0, & \text { otherwise }
\end{array}\right) \\
G_{A^{-}}=G_{A^{-}}\left(u^{(k)}\right)=\operatorname{diag}\left(\begin{array}{ll}
1, & \text { if } u^{(k)}\left(p_{i}\right)<-1 \\
0, & \text { otherwise }
\end{array}\right),
\end{gathered}
$$


where $i=1, \ldots, N$, representing the matrix representations of the generalized derivatives. Note that $M$ is a diagonal symmetric positive definite matrix and $K$ is symmetric and positive semi-definite.

\section{Preconditioning}

For the system matrix in (28), which we write as

$$
A=\left[\begin{array}{cc}
-L & M \\
M & \tau K
\end{array}\right]
$$

we propose the block-triangular preconditioner

$$
P=\left[\begin{array}{cc}
-L & 0 \\
M & -S
\end{array}\right]
$$

Here, $S$ is the Schur complement $\tau K+\tau M L^{-1} M$. Preconditioners of this form have proven to perform well based on the simple observation that the preconditioned matrix $P^{-1} A$ has a small number of distinct eigenvalues. It is obvious that we never want to form the Schur complement explicitly as the storage requirements for realistic scenarios would be not feasible. For $S$ we suggest the following approximation

$$
\hat{S}=\hat{S}_{1} L^{-1} \hat{S}_{2}=(\widehat{M+\sqrt{\tau}} K) L^{-1}(\widehat{M+\sqrt{\tau}} L),
$$

where we use an algebraic multigrid (AMG) preconditioner for the approximation of the inverse of $\hat{S}_{1}$ and $\hat{S}_{2}$. Algebraic multigrid methods typically exhibit geometric multigrid-like properties by only using algebraic information. This has the advantage that it works well in general even for complicated geometries and meshes. We refer to $[41,20]$ for more information on AMG. We also want to emphasize that geometric multigrid (GMG) approximations are also well suited to approximate $\hat{S}_{1}$ and $\hat{S}_{2}$. In the following we want to illustrate the performance of $\hat{S}^{-1} S$. We therefore consider the eigenvalue problem

$$
S v=\lambda \tilde{S} v
$$

with

$$
\tilde{S}=(M+\sqrt{\tau} K) L^{-1}(M+\sqrt{\tau} L)
$$

via Matlab and analyse the robustness of the Schur complement approximation with respect to the penalty parameter $c$ and the mesh parameter $h$. For the former we keep $\varepsilon, \tau$ and $h$ fix and vary $c$ by the sequence 
$c \in\left\{10^{-2}, 10^{-4}, 10^{-6}, 10^{-8}\right\}$ whereas for the latter we fix $\varepsilon, \tau$ and $c$ and vary $h \in\left\{2^{-4}, 2^{-5}, 2^{-6}\right\}$. We choose $\varepsilon$ relative small and set $\tau$ big enough such that $\tau \in\left(0,4 \gamma \varepsilon^{3}\right)$ holds true. For larger $\varepsilon$ we obtain similar results to Figure 5. In the following we present the different types of eigenvalue distributions which we have observed.

We start with the very small choice $\varepsilon=10^{-5}$ and $\tau=4 \cdot 10^{-15}$. For this case we only obtain real eigenvalues.

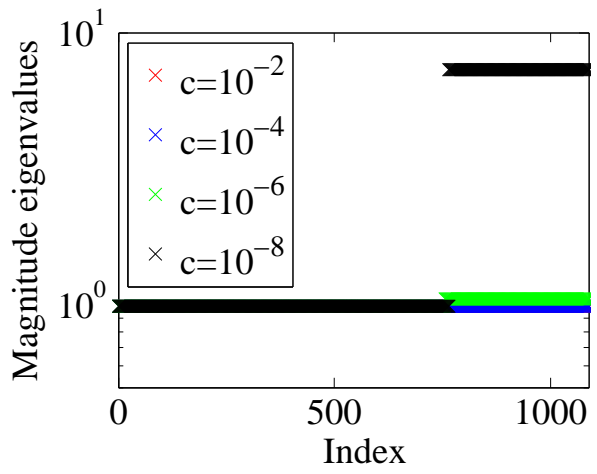

(a) Varying $c$.

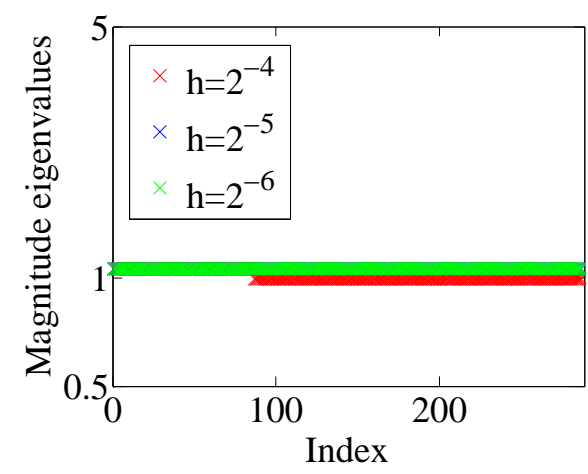

(b) Varying $h$.

Figure 1: Eigenvalues for the Schur complement approximation $(\varepsilon=$ $\left.10^{-5}, \tau=4 \cdot 10^{-15}\right)$.

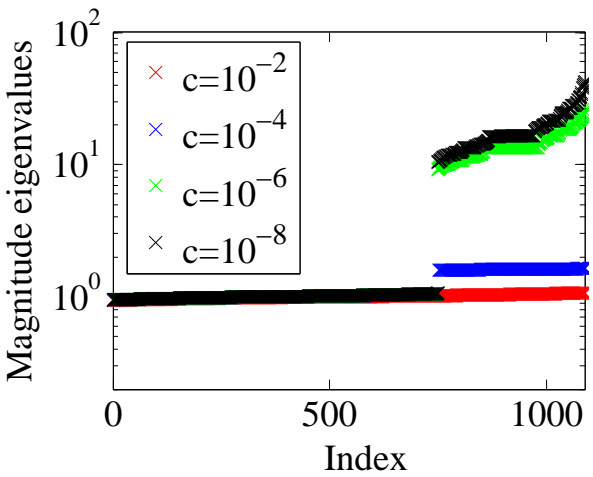

(a) Varying $c$.

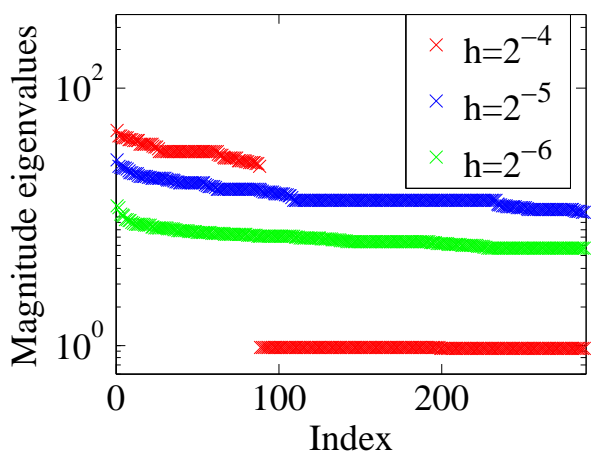

(b) Varying $h$.

Figure 2: Eigenvalues for the Schur complement approximation $(\varepsilon=$ $\left.10^{-3}, \tau=4 \cdot 10^{-9}\right)$. 
In Figure 1 one can clearly see at most two eigenvalue clusters both for varying $c$ and for varying $h$, which are bounded below by one. With slowly increased $\varepsilon$ we get similar eigenvalue distributions, see Figure 2 and [11]. The number of eigenvalue clusters remains constant for varying $c$ and $h$.

Next, we will see that also complex eigenvalues appear, see Figure 3. We set $\varepsilon=6 \cdot 10^{-3}$ and $\tau=8 \cdot 10^{-7}$.

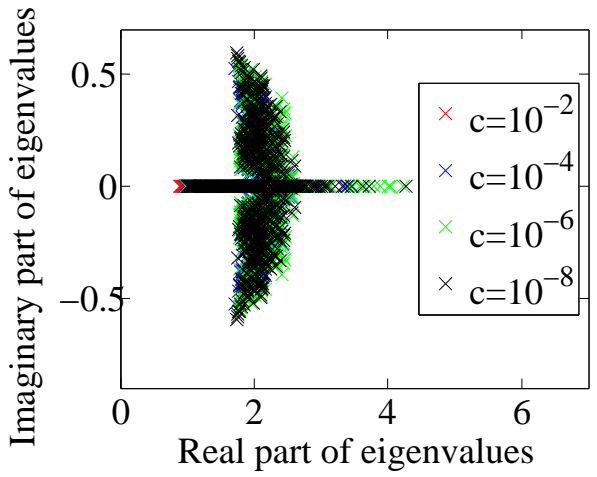

(a) Varying $c$.

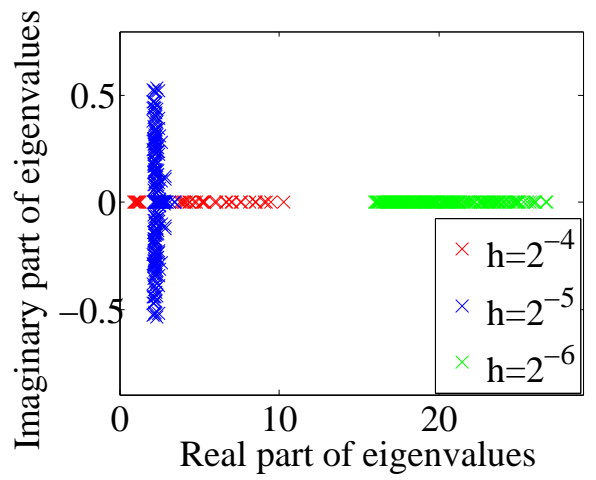

(b) Varying $h$.

Figure 3: Eigenvalues for the Schur complement approximation $(\varepsilon=6$. $\left.10^{-3}, \tau=8 \cdot 10^{-7}\right)$.

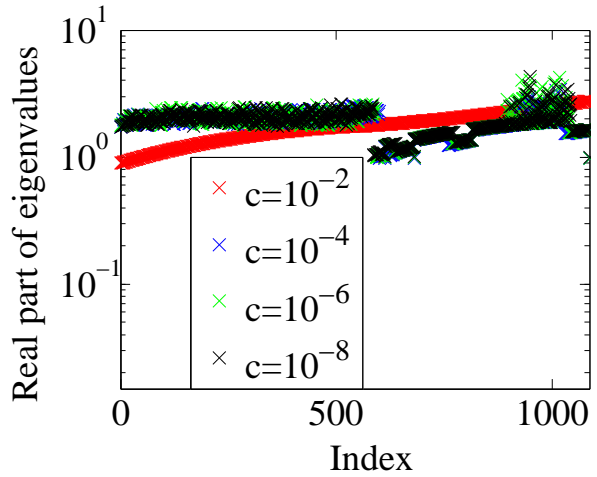

(a) Varying $c$.

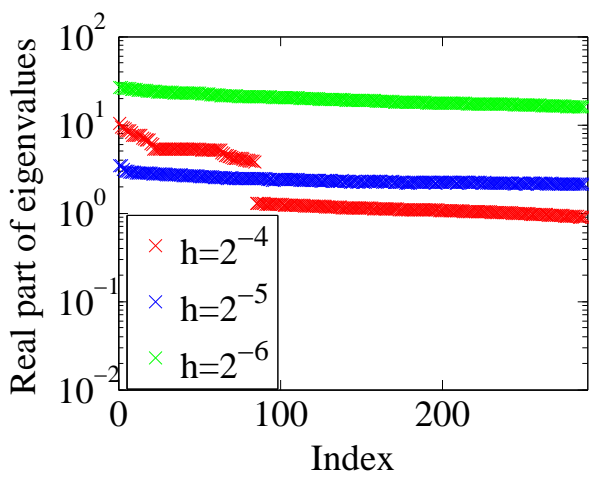

(b) Varying $h$.

Figure 4: Real part of eigenvalues for the Schur complement approximation $\left(\varepsilon=6 \cdot 10^{-3}, \tau=8 \cdot 10^{-7}\right)$. 
For varying $c$ and $h$ the complex eigenvalues are clustered in a circle with radius about 0.5 around the centre 2 . One can see zero imaginary part for the cases $h \in\left\{2^{-4}, 2^{-6}\right\}$ on the right. The real parts are considered separately in Figure 4. In Figure 4(a), we plot the real part of the eigenvalues of Figure 3 (a) and observe (possibly except for the case $c=10^{-2}$ ) almost the same clustering for varying $c$. More precisely, we have a cluster around 1 and 2 . The real parts with respect to Figure 3(b) are considered in Figure 4(b). Here, we have different eigenvalues for varying $h$, but the small number of clusters stays almost constant.

Finally, at a certain $\varepsilon$, we get very good results for the Schur complement approximation because there appears only one eigenvalue cluster around 1 . In Figure 5, we show such an example for the choice of $\varepsilon=2 \cdot 10^{-1}$ and $\tau=3 \cdot 10^{-2}$. Except for only a few outliers there is just one eigenvalue cluster around 1 independent of $c$ and $h$.

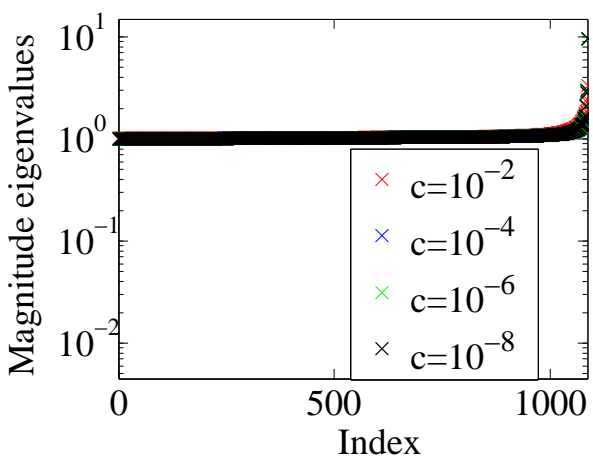

(a) Varying $c$.

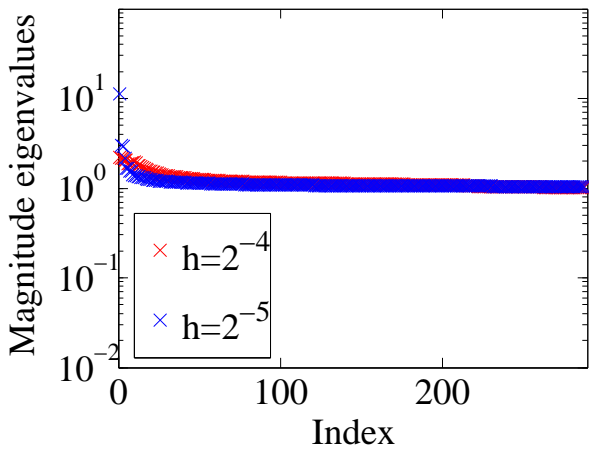

(b) Varying $h$.

Figure 5: Eigenvalues for the Schur complement approximation $(\varepsilon=2$. $\left.10^{-1}, \tau=3 \cdot 10^{-2}\right)$.

We have seen that we obtained different eigenvalue distributions for several examples. However, we also observed only a small number of eigenvalue clusters, which justifies our choice of $\hat{S}$.

For the application of $P$ it remains to analyse the computational solving of a linear system with the block $L$. The aim of a preconditioner is to resemble the original matrix by also being easy to invert. Hence, we need to approximate the matrix $L$ efficiently. A typical approximation for $L$ would be the use of a multigrid method of geometric (GMG) $[47,28]$ or algebraic 
nature (AMG) [41, 20]. Similar to our Schur complement approximation we focus here on the use of algebraic multigrid. More precisely, for all AMG preconditioners in $P$ we use the smoothed aggregation AMG implemented as part of the Trilinos [29] ML package [24]. The ML package allows for a variety of smoothers and in our experiences 10 steps of the Chebyshev smoother were superior to similar or smaller numbers of Gauss-Seidel or Jacobi smoothing steps. The Chebyshev smoother is the recommended option for the discretization of elliptic operators, see e.g. [29]. The use of two AMG $\mathrm{V}$-cycles was always sufficient for our experiments and in fact we did not observe improvements when a larger number of $\mathrm{V}$-cycles was used.

The matrix $L=\gamma \varepsilon K+\frac{1}{c} G_{A} M G_{A}-\frac{1}{\varepsilon} M$ can easily become indefinite. In this case, $L$ is similar to a discrete Helmholtz operator. In [19] it is described how difficult it is to solve Helmholtz problems with classical iterative methods. For this reason and also because of the demand of a positive definite preconditioner for symmetric Krylov subspace methods we switch to the non-symmetric system matrix

$$
\tilde{A}=\left[\begin{array}{cc}
M & -L \\
\tau K & M
\end{array}\right]
$$

Note that as the block $L$ is indefinite we would have to use non-symmetric iterative methods anyway. As before, we propose the block-triangular preconditioner

$$
\tilde{P}=\left[\begin{array}{cc}
M & 0 \\
\tau K & -S
\end{array}\right]
$$

For the Schur complement $M+\tau K M^{-1} L$ we use a similar approximation as for system (29):

$$
\hat{S}=\hat{S}_{1} M^{-1} \hat{S}_{2}=(\widehat{M+\sqrt{\tau}} K) M^{-1}(\widehat{M+\sqrt{\tau}} L) .
$$

Note, that we not need to solve a linear system with the difficult block $L$ anymore because the approximation of $M+\sqrt{\tau} L$ reduces the influence of the indefiniteness of $L$. More precisely, the positive definiteness of $M+\sqrt{\tau} L$ is guaranteed for $\tau<\varepsilon^{2}$. Finally, we also would like to state explicitly that we can use the same preconditioning strategy for the case of the semi-implicit Cahn-Hilliard model. In this case, we have the preconditioner

$$
P_{s i}=\left[\begin{array}{cc}
-L_{s i} & 0 \\
M & -S
\end{array}\right]=\left[\begin{array}{cc}
-\gamma \varepsilon K-\frac{1}{c} G_{A} M G_{A} & 0 \\
M & -S
\end{array}\right],
$$


where we suggest the Schur complement approximation

$$
\hat{S}=\hat{S}_{1} L_{s i}^{-1} \hat{S}_{2}=(\widehat{M+\sqrt{\tau}} K) L_{s i}^{-1}\left(M \widehat{+\sqrt{\tau}} L_{s i}\right),
$$

as well as

$$
\hat{L}_{s i}=\left\{\begin{aligned}
\widehat{L_{s i}+\varepsilon \tau} I, & \text { if } G_{A}=0 \\
\widehat{L_{s i}}, & \text { otherwise }
\end{aligned}\right.
$$

with $I$ being the identity matrix. With the approximation of $\hat{L}_{s i}$ in (31) we solve the problem if $L_{s i}$ becomes the pure Neumann-Laplace operator, where the shift $\varepsilon \tau$ is an heuristic choice. For both time discretizations we obtain very fast convergence rates, see Section 8.

\section{Numerical results}

In this section we show results for the Cahn-Hilliard problem. The preconditioners we presented can be embedded into various Krylov subspace solvers. For the non-symmetric matrix (30) we propose the use of a nonsymmetric short-term recurrence method, namely BiCG [21], but note that also other solvers such as QMR [22], BiCGSTAB [46] or GMRES [43] can be used with this preconditioner. For the numerical results presented in this section we choose the BiCG tolerance to be $10^{-7}$ for the preconditioned residual in all examples. For the multilevel approximations we choose Trilinos AMG approximations [29]. For one application of the preconditioner we take in general 10 steps of a Chebyshev smoother and two V-cycles. The discretization is performed with deal.II [1], which allows the use of the Trilinos library. All numerical experiments listed here are generated with finite elements on rectangles. For the semi-smooth Newton method we use the stopping criterion in [31], given by

$$
\left\|F_{c}\left(u_{h}^{(k)}, w_{h}^{(k)}\right)\right\|_{2} \leq \epsilon_{\mathrm{rel}}\left\|F_{c}\left(u_{h}^{(0)}, w_{h}^{(0)}\right)\right\|_{2}+\epsilon_{\mathrm{abs}}, \quad k=1, \ldots, k_{\max },
$$

where we set $k_{\max }=100, \epsilon_{\mathrm{rel}}=10^{-12}$ and $\epsilon_{\mathrm{abs}}=10^{-6}$ in all examples. For the handling of the parameter $c$ we follow [31] and solve (24)-(25) for the sequence $c_{1}=10^{-1} \geq c_{2}=10^{-2} \geq \ldots \geq c_{\max }$, where we initialize the Newton methods by the approximate solutions of the previous ones respectively. After a few time steps (typically 1 time step), we fix $c=c_{\max }$. This is because the initial solutions at the beginning might not be a good starting point for the semi-smooth Newton methods. 


\subsection{Mesh adaptation}

To generate the adaptive meshes we use a mesh adaptation strategy similar to Blank et al. [5]. Experiments show that it is essential to ensure that at least eight vertices lie on the interfaces to avoid mesh effects, see also [10]. We hence refine the interface to a level where eight vertices are within the interface and coarse in areas where the concentration $u$ is constant. For given parameters $\varepsilon$ and $\gamma$ this results in an upper bound $h_{\min } \leq \varepsilon \sqrt{\gamma} \frac{\pi}{9}$, where $h_{\text {min }}$ is the refinement level on the interface. Since we want to avoid meshes which are too coarse, we additionally define $h_{\max }:=10 \cdot h_{\min }$. Afterwards the mesh adaptation is done using the following strategy: An element $R \in R_{h}$ is marked for refinement if it satisfies $h_{R}>h_{\min }$ and if it, or one of its neighboring elements, satisfies $|u(x)|<1$ for some $x$. Here $x$ can be a node or the middle point of a cell. An element $R \in R_{h}$ is marked for coarsening, if it satisfies $h_{R}<h_{\max }$ and $|u(x)| \geq 1$ for all $x$. Thereby, we refine in an area, which contains the interface and coarsen within the pure phases. Since we also include the neighboring cells for the refinement process, we do not coarsen too close to the interface. Note that it is also possible to incorporate other refinement strategies such as [31].

\subsection{Comparison of implicit and semi-implicit time discretization}

As in [13], we consider $\Omega=B_{1}(0) \subset \mathbb{R}^{2}$ and two circles around 0 with radii $r_{1}=0.3$ and $r_{2}=0.15$ as initial values.

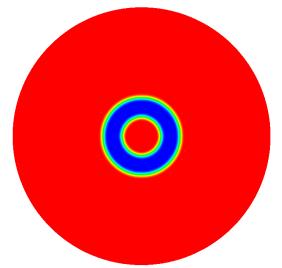

(a) $t=10^{-5}$

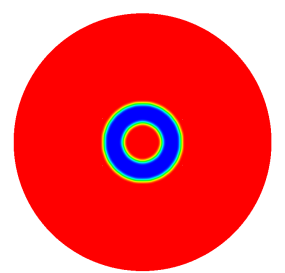

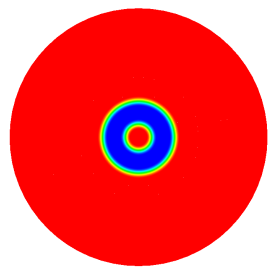

(b) $t=10^{-3}$

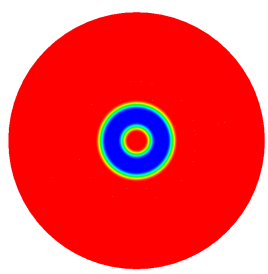

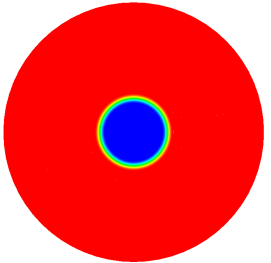

(c) $t=1.85 \cdot 10^{-3}$

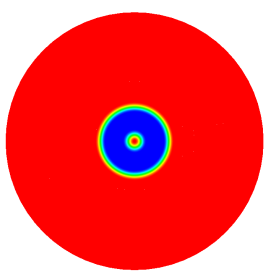

Figure 6: Implicit (above) and semi-implicit (below) Cahn-Hilliard evolution for the circle computation. 
The time evolution of the exact solution to the Mullins-Sekerka model results in a shrinking of both radii until the smaller one vanishes at time $t_{c}=$ $1.85 \cdot 10^{-3}$. We want to compare the evolution of the initial data for the implicit and semi-implicit Cahn-Hilliard model with respect to the step size parameter $\tau$. For $\varepsilon=0.025$ and $\tau=10^{-5}$, where $\tau<4 \gamma \varepsilon^{3}$ holds true, we see in Figure 6, that in the semi-implicit simulation the smaller circle vanishes later in time than in the more precise implicit case. Now we explore the evolution of the semi-implicit system for larger $\tau$, as this case has no theoretical time-step restriction. We choose $\varepsilon=0.02$ and vary $\tau$. In Figure 7 , we consider the solutions at time $t=3 \cdot 10^{-3}$, where for the exact solution the smaller circle has already vanished.

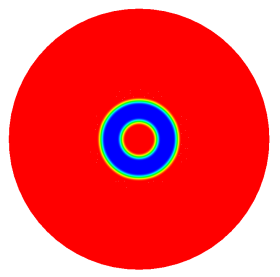

(a) $\tau=5 \cdot 10^{-4}$

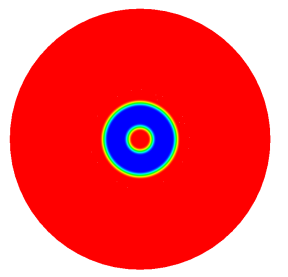

(b) $\tau=1 \cdot 10^{-4}$

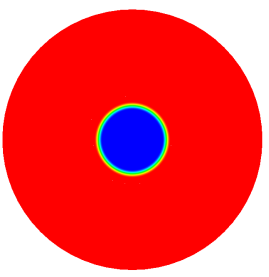

(c) $\tau=3 \cdot 10^{-5}$

Figure 7: Semi-implicit Cahn-Hilliard evolution for variable $\tau$.

In Figure 7(a) and $7(\mathrm{~b})$ we have $\tau>4 \gamma \varepsilon^{3}$. We can see that the approximation is crude for larger time steps and very small time steps are necessary to capture the evolution of the sharp interface model. This example verifies our preference for the implicit time discretization despite the time-step restriction.

\subsection{One-dimensional example}

As in [31], we test the validity and performance of the Moreau-Yosida semi-smooth Newton solver. From the initial data

$$
u^{(0)}(x)=\left\{\begin{array}{cl}
\cos \left(\frac{x-\frac{1}{2}}{\varepsilon}\right)-1, & \text { if }\left|x-\frac{1}{2}\right| \leq \frac{1}{2} \pi \varepsilon \\
-1, & \text { otherwise }
\end{array}\right.
$$

we want to recover the exact stationary one-dimensional solution

$$
u_{\mathrm{ex}}(x)=\left\{\begin{aligned}
\frac{1}{\pi}\left[1+\cos \left(\frac{x-\frac{1}{2}}{\varepsilon}\right)\right]-1, & \text { if }\left|x-\frac{1}{2}\right| \leq \pi \varepsilon \\
-1, & \text { otherwise }
\end{aligned}\right.
$$


see $[2,8]$. We choose $\Omega=(0,1), \varepsilon=0.1$ as well as $\tau=0.001$ and vary $c_{\max }$ and $h$ for uniform meshes. For the handling of the parameter $c$, we use the $c$-sequence (described in the beginning of this section) for the first three time steps. Then, we fix $c=c_{\max }$. The simulations are stopped as soon as $\left\|u_{c, h}(t)-u_{c, h}(t-\tau)\right\|_{2} \leq 10^{-7}$.

\begin{tabular}{ccccc}
\hline & \multicolumn{3}{c}{ max SSN $\backslash$ BiCG } \\
$h$ & $c_{\max }$ & $\left\|u_{c_{\max }, h}-u_{\mathrm{ex}, h}\right\|_{2}$ & iterations & CPU time $(\mathrm{s})$ \\
\hline$\frac{1}{2^{6}}$ & $10^{-3}$ & $2.46337 \cdot 10^{-2}$ & $2 \backslash 14$ & 0.34 \\
& $10^{-6}$ & $3.05118 \cdot 10^{-3}$ & $2 \backslash 14$ & 0.41 \\
& $10^{-9}$ & $3.06076 \cdot 10^{-3}$ & $2 \backslash 14$ & 0.50 \\
$\frac{1}{2^{7}}$ & $10^{-3}$ & $3.58987 \cdot 10^{-2}$ & $3 \backslash 15$ & 0.61 \\
& $10^{-6}$ & $9.32589 \cdot 10^{-4}$ & $3 \backslash 16$ & 0.87 \\
& $10^{-9}$ & $9.31919 \cdot 10^{-4}$ & $3 \backslash 16$ & 1.00 \\
$\frac{1}{2^{8}}$ & $10^{-3}$ & $5.04977 \cdot 10^{-2}$ & $3 \backslash 15$ & 1.17 \\
& $10^{-6}$ & $6.92492 \cdot 10^{-4}$ & $3 \backslash 16$ & 1.80 \\
& $10^{-9}$ & $6.71485 \cdot 10^{-4}$ & $3 \backslash 17$ & 2.12 \\
$\frac{1}{2^{9}}$ & $10^{-3}$ & $7.10723 \cdot 10^{-2}$ & $3 \backslash 15$ & 2.17 \\
& $10^{-6}$ & $2.63167 \cdot 10^{-4}$ & $3 \backslash 16$ & 3.55 \\
& $10^{-9}$ & $1.99167 \cdot 10^{-4}$ & $3 \backslash 17$ & 4.07 \\
\hline
\end{tabular}

Table 1: l2-error of the computed solutions as well as maximal number of SSN and BiCG iterations for different mesh sizes and penalty parameters.

In Table 1, we show the maximal number of SSN and BiCG iterations, the error between the exact and the computed solution, and the total time for the computation. We can see the (almost) mesh-independent convergence behaviour for BiCG as well as for the SSN method. The number of SSN and $\mathrm{BiCG}$ iterations does not exceed 3 and 17, respectively, regardless of the mesh size or the chosen penalty parameter.

\subsection{Spinodal decomposition in 2D}

The next example starts with random data in two space dimensions where we set the initial value $u^{(0)}(x)$ randomly between -0.3 and 0.5 , i.e. no pure phases are present at time $t=0$. The domain is set to be $\Omega=(-1,1)^{2}$. Figure 8 shows the initial configuration and the result of the Cahn-Hilliard evolution after 5,50, and 500 time steps. For this computation we use an adaptively refined and coarsened mesh with the minimal size of an element $h_{\min }=\frac{\varepsilon \sqrt{\gamma} \pi}{9}$ and the maximal size $h_{\max }=10 \cdot h_{\min }$ as well as $h_{0}=\frac{2}{64}, \varepsilon=$ $0.02, \tau=10^{-5}$ and $c=c_{\max }=10^{-7}$. 


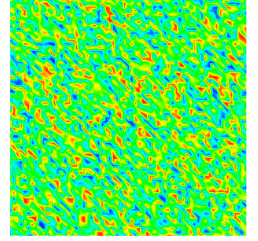

(a) $t=0$

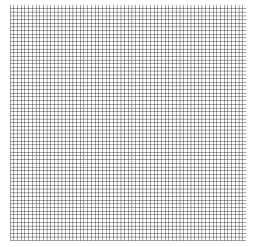

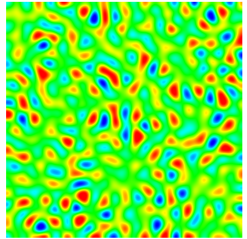

(b) $t=5 \tau$

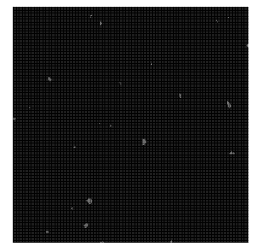

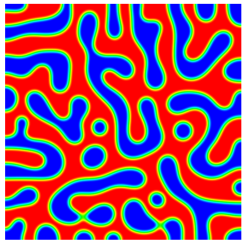

(c) $t=50 \tau$

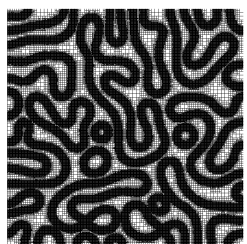

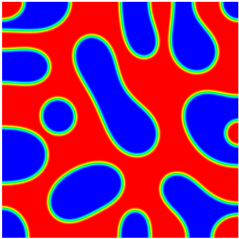

(d) $t=500 \tau$

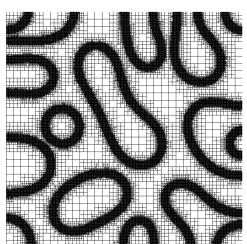

Figure 8: Computation with random initial data.

\begin{tabular}{crcc}
\hline time step & dimension & SSN & BiCG per SSN \\
\hline 0 & 4225 & 1 & 24 \\
1 & 16641 & 1 & 28 \\
2 & 66049 & 1 & 27 \\
3 & 263169 & 7 & 22 \\
4 & 263125 & 7 & 58 \\
5 & 262702 & 7 & 66 \\
6 & 260663 & 7 & 53 \\
7 & 254934 & 7 & 40 \\
8 & 246062 & 7 & 33 \\
9 & 238645 & 7 & 31 \\
10 & 233610 & 6 & 30 \\
11 & 230127 & 7 & 28 \\
12 & 226956 & 6 & 30 \\
13 & 224212 & 6 & 27 \\
14 & 221693 & 6 & 27 \\
15 & 219844 & 6 & 27 \\
16 & 217745 & 5 & 26 \\
17 & 215950 & 6 & 25 \\
18 & 214211 & 6 & 26 \\
19 & 212293 & 5 & 26 \\
\hline
\end{tabular}

Table 2: Number of SSN and BiCG iterations for the spinodal decomposition with adaptive mesh. 
Table 2 shows the average number of BiCG iterations per Newton iteration for the first 20 time steps. We also use uniform mesh sizes and compare the average number of BiCG iterations per Newton iteration as well as the number of Newton iterations per time step, see Figure 9. In the Figure the number of unknowns is listed. It can be seen that at the beginning of the computation when no pure phases are present the preconditioning and Newton method work very well. Then we observe a very benign growth. However the number of iterations stay low with at most 40 iterations for a mesh with roughly one million unknowns.

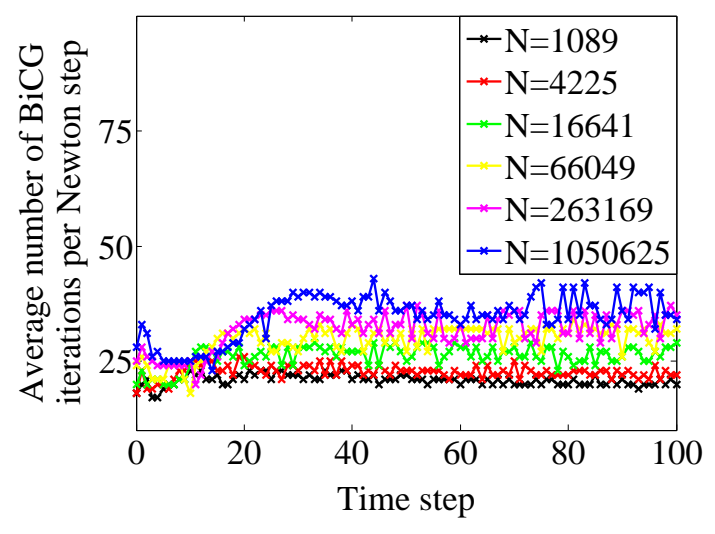

(a) $\mathrm{BiCG}$

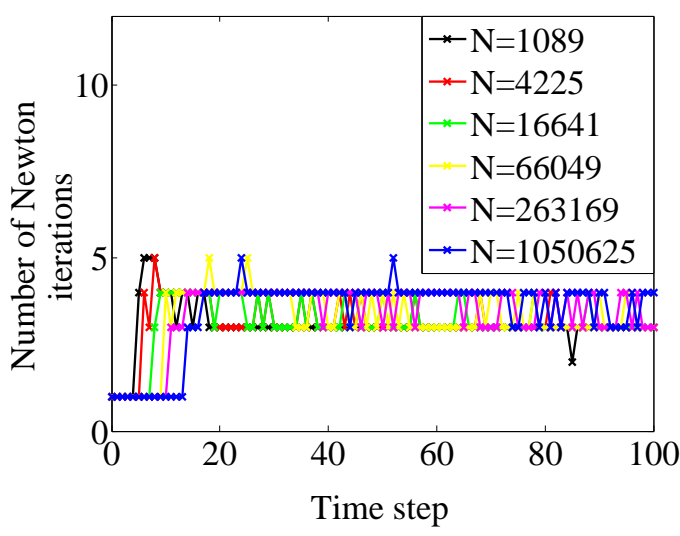

(b) Newton

Figure 9: Results for 100 time steps of a random initial data with different uniform mesh sizes.

\subsection{Two-dimensional square}

In the next example we set $\Omega=\left(-\frac{1}{2}, \frac{1}{2}\right)$ and compute the evolution of the unit square shown in Figure 10, see also [4]. For this computation we choose an adaptive mesh with $h_{\min }=\frac{\varepsilon \sqrt{\gamma} \pi}{9}, h_{\max }=10 \cdot h_{\min }, h_{0}=\frac{1}{2^{7}}$ as well as $\varepsilon=\frac{1}{12 \pi}, \tau=10^{-5}$ and $c_{\max }=10^{-7}$ with $c=c_{\max }$ fix for $t>0$. 


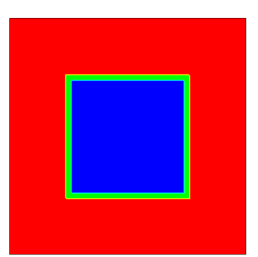

(a) $t=0 \tau$

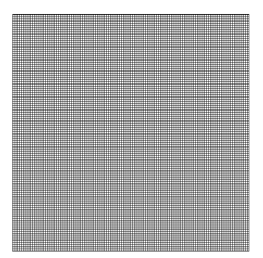

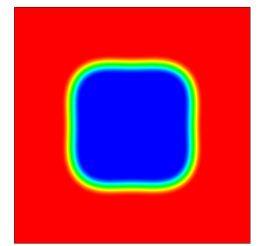

(b) $t=20 \tau$

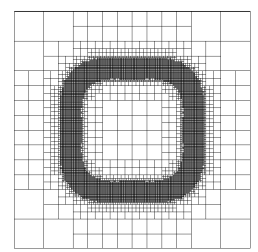

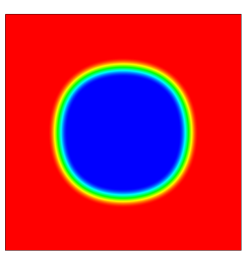

(c) $t=80 \tau$

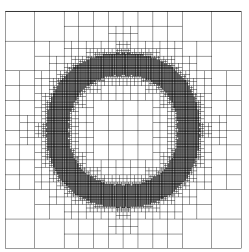

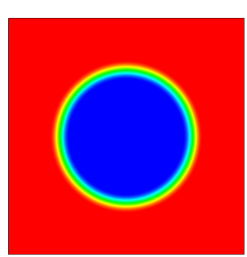

(d) $t=280 \tau$

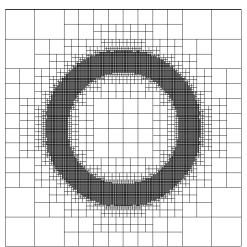

Figure 10: Evolution of a square to a circle.

First we compute the solution for a sequence of uniform meshes with $h=\frac{1}{2^{l} 128}$ for $l=0,1,2$ and $\varepsilon=\frac{1}{12 \pi}, \tau=10^{-5}, T=2 \cdot 10^{-4}$ as well as $c_{\text {max }}=10^{-7}$ with $c=c_{\max }$ fix for $t>0$. In Figure 11, we show the average number of BiCG iterations per Newton step as well as the number of SSN iterations per time step, which stay (almost) constant for one mesh size.

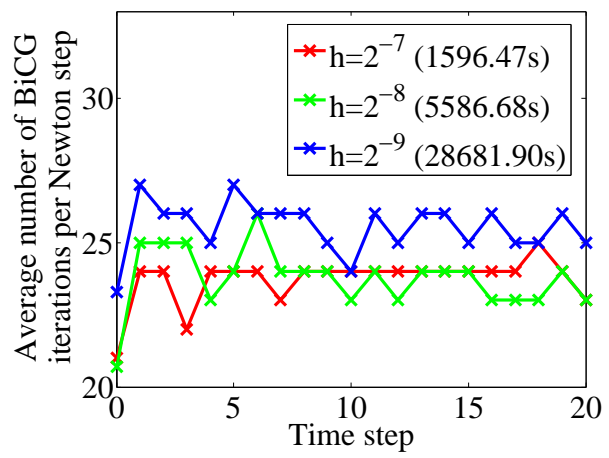

(a) BiCG

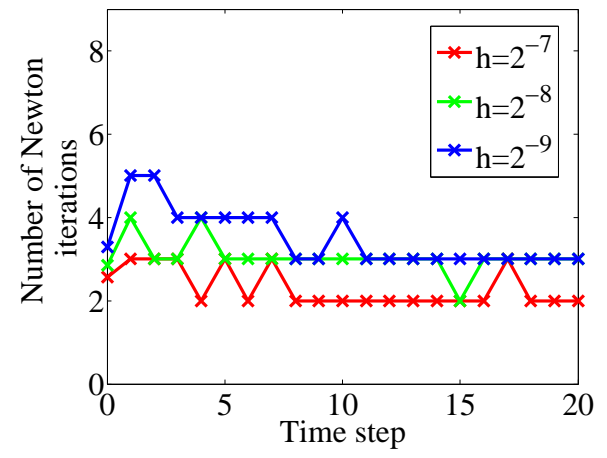

(b) Newton

Figure 11: Square computation: 20 time steps for different uniform mesh sizes.

We also investigate the convergence of BiCG and SSN on adaptive meshes 
with $h_{0}=\frac{1}{2^{7}}, h_{\min }=\frac{1}{2^{l} 128}$ and $h_{\max }=\frac{1}{2^{l}}$ for $l=0,1,2$. Figure 12 shows again that the number of SSN iterations and average number of $\mathrm{BiCG}$ iterations remain (almost) constant for different mesh sizes.

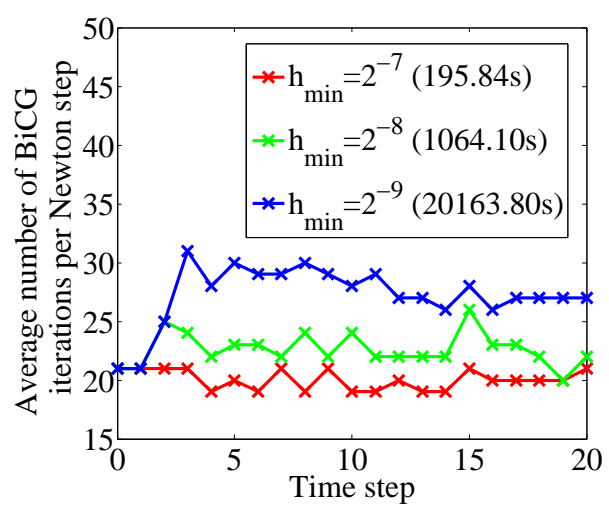

(a) BiCG

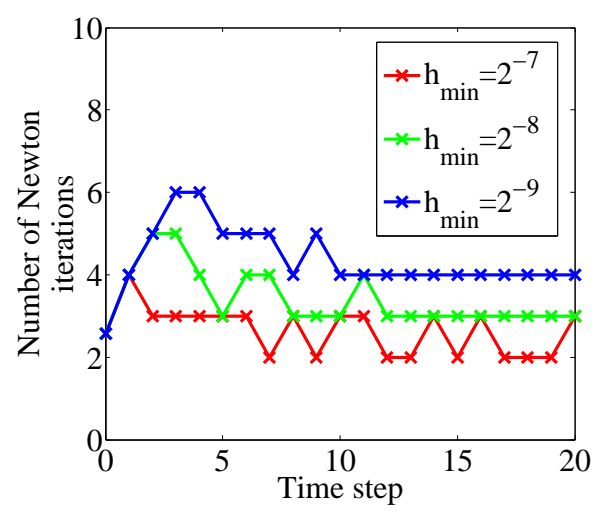

(b) Newton

Figure 12: Square computation: 20 time steps for adaptive meshes.

\subsection{Three-dimensional dumbbell}

In the last computation we consider the three dimensional domain $\Omega=$ $(-1,1)^{3}$ and choose a dumbbell as initial state.

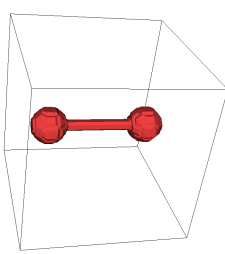

(a) $t=0 \tau$

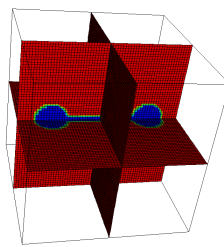

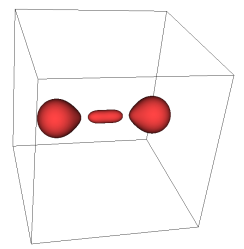

(b) $t=4 \tau$

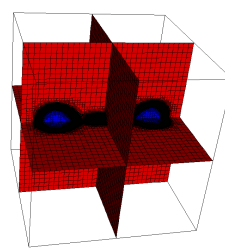

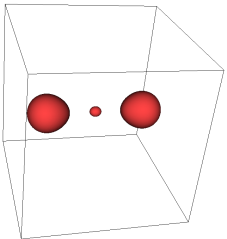

(c) $t=7 \tau$

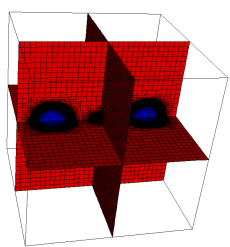

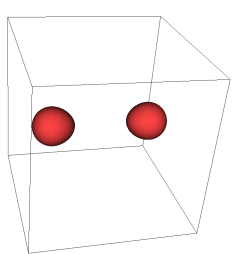

(d) $t=8 \tau$

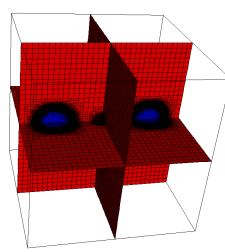

Figure 13: Evolution of a dumbbell. 
Figure 13 shows the evolution for this example. For this computation we use an adaptive mesh with $h_{0}=\frac{2}{2^{6}}, h_{\min }=\frac{\varepsilon \sqrt{\gamma} \pi}{9}, h_{\max }=10 \cdot h_{\min }$ as well as $\varepsilon=0.03, \tau=5 \cdot 10^{-5}$ and $c_{\max }=10^{-5}$ with $c=c_{\max }$ fix for $t>0$. Table 2 shows the average number of BiCG iterations per Newton iteration for the first 15 time steps.

\begin{tabular}{crcc}
\hline time step & dimension & SSN & BiCG per SSN \\
\hline 0 & 274625 & 5 & 24 \\
1 & 108797 & 4 & 42 \\
2 & 485773 & 6 & 45 \\
3 & 2929083 & 10 & 52 \\
4 & 2642293 & 10 & 50 \\
5 & 2681443 & 10 & 50 \\
6 & 2526021 & 9 & 48 \\
7 & 2512839 & 4 & 52 \\
8 & 2368893 & 4 & 47 \\
9 & 2421035 & 3 & 49 \\
10 & 2354253 & 3 & 47 \\
11 & 2417651 & 3 & 48 \\
12 & 2358727 & 3 & 45 \\
13 & 2415291 & 3 & 46 \\
14 & 2360207 & 3 & 46 \\
\hline
\end{tabular}

Table 3: Number of SSN and BiCG iterations for the dumbbell with adaptive mesh.

\section{Other available solvers}

In this section, we compare our solution technique to existing solution methods. Traditionally iterative solvers such as (nonlinear) Gauss-Seidel have been used for the smooth and non-smooth case, but these suffer from deteriorating convergence rates for increasing refinement [18, 3, 37].

In [25] Gräser and Kornhuber proposed a preconditioned Uzawa iteration method for the saddle point formulation of the discrete (semi-implicit in time) Cahn-Hilliard system with an obstacle potential [26]. The method proceeds as follows. Consider the $k$-th Uzawa iteration which consists of two sub-steps: At first, an elliptic obstacle problem with box constraints needs to be solved to obtain $u^{(k)}$ and the appropriate coincidence set

$$
\mathcal{N}_{h}^{*}\left(u^{(k)}\right)=\left\{p \in J_{h}:\left|u^{(k)}(p)\right|=1\right\} .
$$


With the help of this set, the second sub-step reduces to a linear saddle point problem, which has to be solved for getting $w^{(k+1)}$. Once the exact coincidence set $\mathcal{N}_{h}^{*}(u)$ is detected, the Uzawa iteration provides the exact solution (for the considered time step). Gräser and Kornhuber apply a monotone multigrid method [36] to the first subproblem and a multigrid method with a block Gauss-Seidel smoother to the second one. Although their algorithm exhibits superior convergence properties to traditional stationary iterative solvers, they cannot guarantee mesh-independence. Particularly, first numerical evidence suggests an increase in the number of iterations for refined meshes, see e.g. [26], which is not the case for our method.

Baňas and Nürnberg propose a full nonlinear multigrid method for the discrete Cahn-Hilliard problem. For a sequence of triangulations $\mathcal{T}_{k}$, the algorithm consists of alternating pre-smoothing steps for the approximate solution by projected Gauss-Seidel iterations [3] and restrictions to the next coarser grid. On the coarsest triangulation the appropriate system is solved exactly. By prolongation to the next finer grid, the solution is updated together with post-smoothing steps by projected Gauss-Seidel iterations. This method exhibits mesh-independent convergence properties in practice for arbitrary time steps in 2D and 3D and also for a small interfacial parameter $\varepsilon$. However, Baňas and Nürnberg are unable to prove convergence of the multigrid solver (except for the case, when the discrete Cahn-Hilliard system reduces to a linear problem, which occurs when $\left|u^{(n)}\right|<1$ ). The same holds for methods including a smooth potential, see [34, 35]. In contrast, we are able to prove convergence.

A block preconditioning strategy is proposed by Neytcheva et al. [12]. They consider the Cahn-Hilliard equation with a smooth potential coupled with the Navier-Stokes equation and apply two fully implicit schemes of the $\theta$-method (backward Euler and Crank-Nicolson) for the discretization in time. This leads to solving nonlinear systems at each time step for which they apply Newton's method. Each Newton step involves a solution of a non-symmetric linear system with the Jacobian matrix. By simplifying the system matrix they get a preconditioner, which is proven to yield optimal results. Neytcheva et al. show that their preconditioning technique is superior over a fast sparse direct solver and also shows robust convergence behaviour. Contrary to us, they do not consider non-smooth potentials. 


\section{Conclusions}

In this paper we have analysed the linear systems arising from the semismooth Newton method for the Moreau-Yosida regularized Cahn-Hilliard equation. Additionally, we have shown superlinear convergence of the SSN method in function space for the implicit time discretized problem. In order to make the SSN method more efficient we have used a Krylov subspace solver. We have introduced and studied block-triangular preconditioners using an efficient and cheap Schur complement approximation. This approximation can be done using multilevel techniques, algebraic multigrid in our case, and the numerical results justify this choice. Note that the same preconditioning techniques can be applied to the semi-implicit discretized Cahn-Hilliard problem.

\section{Acknowledgements}

Parts of this work were performed while the first author was visiting the Oxford Center for Collaborative Applied Mathematics (OCCAM), University of Oxford. This publication was based on work supported in part by Award No. KUK-C1-013-04, made by King Abdullah University of Science and Technology (KAUST). The authors would like to thank Christian Kahle and Michael Hinze for their comments.

\section{References}

[1] W. Bangerth, R. Hartmann, and G. Kanschat, deal.II - a general purpose object oriented finite element library, ACM Trans. Math. Softw., 33 (2007), pp. 24/1-24/27.

[2] J. W. Barrett, J. F. Blowey, and H. Garcke, Finite element approximation of the Cahn-Hilliard equation with degenerate mobility, SIAM J. Numer. Anal., 37 (1999), pp. 286-318.

[3] J. W. Barrett, R. Nürnberg, and V. Styles, Finite element approximation of a phase field model for void electromigration, SIAM J. Numer. Anal., 42 (2004), pp. 738-772.

[4] L. Bañas And R. Nürnberg, A multigrid method for the CahnHilliard equation with obstacle potential, Appl. Math. Comput., 213 (2009), pp. 290-303. 
[5] L. Blank, M. Butz, And H. Garcke, Solving the Cahn-Hilliard variational inequality with a semi-smooth Newton method, ESAIM Control Optim. Calc. Var., 17 (2011), pp. 931-954.

[6] L. Blank, H. Garcke, L. Sarbu, and V. Styles, Primal-dual active set methods for Allen-Cahn variational inequalities with nonlocal constraints, Numer. Methods Partial Differ. Equ., (2012).

[7] L. Blank, L. Sarbu, And M. Stoll, Preconditioning for Allen-Cahn variational inequalities with non-local constraints, J. Comput. Phys., 231 (2012), pp. 5406-5420.

[8] J. F. Blowey and C. M. Elliott, The Cahn-Hilliard gradient theory for phase separation with non-smooth free energy Part I: Mathematical analysis, Eur. J. Appl. Math., 2 (1991), pp. 233-280.

[9] — The Cahn-Hilliard gradient theory for phase separation with nonsmooth free energy Part II: Numerical analysis, Eur. J. Appl. Math., 3 (1992), pp. 147-179.

[10] _ Curvature dependent phase boundary motion and parabolic double obstacle problems, in Degenerate diffusions (Minneapolis, MN, 1991), vol. 47 of IMA Vol. Math. Appl., Springer, New York, 1993, pp. 19-60.

[11] J. Bosch, Fast solvers for Cahn-Hilliard variational inequalities (in German), Master's thesis, Universität Magdeburg, September 2012.

[12] P. Boyanova, M. Do-Quang, and M. Neytcheva, Efficient preconditioners for large scale binary Cahn-Hilliard models, Comput. Methods Appl. Math., 12 (2012), pp. 1-22.

[13] M. Butz, Computational methods for Cahn-Hilliard variational inequalities, PhD thesis, Universität Regensburg, March 2012.

[14] J. W. Cahn And J. E. Hilliard, Free Energy of a Nonuniform System. I. Interfacial Free Energy, J. Chem. Phys., 28 (1958), pp. 258-267.

[15] M. I. M. Copetti And C. M. Elliott, Numerical analysis of the Cahn-Hilliard equation with a logarithmic free energy, Numer. Math., 63 (1992), pp. 39-65. 
[16] T. A. DAVIS, UMFPACK version 4.4 user guide, tech. rep., Dept. of Computer and Information Science and Engineering Univ. of Florida, Gainesville, FL, 2005.

[17] I. C. Dolcetta, S. F. Vita, And R. March, Area-preserving curveshortening flows: From phase separation to image processing, Interfaces Free Bound., 4 (2002), pp. 325-343.

[18] C. M. Elliott, The Cahn-Hilliard model for the kinetics of phase separation, in Mathematical Models for Phase Change Problems, J. F. Rodrigues, ed., vol. 88 of Int. Ser. Numer. Math., Birkhäuser, Basel, 1989, pp. 35-73.

[19] O. G. ERnst And M. J. Gander, Why it is difficult to solve Helmholtz problems with classical iterative methods, in Numerical Analysis of Multiscale Problems, I. G. Graham, T. Y. Hou, O. Lakkis, and R. Scheichl, eds., vol. 83 of Lect. Notes Comput. Sci. Eng., Springer Berlin Heidelberg, 2012, pp. 325-363.

[20] R. D. Falgout, An Introduction to Algebraic Multigrid, Comput. Sci. Eng., 8 (2006), pp. 24-33. Special Issue on Multigrid Computing.

[21] R. FletCHER, Conjugate gradient methods for indefinite systems, in Numerical Analysis, G. A. Watson, ed., vol. 506 of Lect. Notes in Math., Springer Berlin Heidelberg, 1976, pp. 73-89.

[22] R. W. FReund AND N. M. NACHTIGAL, QMR: a quasi-minimal residual method for non-Hermitian linear systems, Numer. Math., 60 (1991), pp. 315-339.

[23] H. Garcke, Mechanical effects in the Cahn-Hilliard model: A review on mathematical results, in Mathematical methods and models in phase transitions, A. Miranville, ed., Nova Science Publishers, New York, 2005, pp. $43-77$.

[24] M. W. Gee, C. M. Siefert, J. J. Hu, R. S. Tuminaro, and M. G. SAlA, ML 5.0 smoothed aggregation user's guide, Tech. Rep. SAND2006-2649, Sandia National Laboratories, 2006. 
[25] Glowinski, R. And Lions, J. L. and Trémolières, R., ed., $N u$ merical Analysis of Variational Inequalities, vol. 8 of Stud. Math. Appl., Elsevier, 1981.

[26] C. Gräser And R. Kornhuber, On preconditioned Uzawa-type iterations for a saddle point problem with inequality constraints, in Domain Decomposition Methods in Science and Engineering XVI, O. B. Widlund and D. E. Keyes, eds., vol. 55 of Lect. Notes Comput. Sci. Eng., Springer Berlin Heidelberg, 2007, pp. 91-102.

[27] A. Greenbaum, Iterative methods for solving linear systems, vol. 17 of Frontiers in Applied Mathematics, SIAM, Philadelphia, PA, 1997.

[28] W. HackBusch, Multigrid methods and applications, vol. 4 of Springer Ser. Comput. Math., Springer-Verlag, Berlin, 1985.

[29] M. Heroux, R. Bartlett, V. H. R. Hoekstra, J. Hu, T. Kolda, R. Lehoucq, K. Long, R. Pawlowski, E. Phipps, A. Salinger, H. Thornquist, R. Tuminaro, J. Willenbring, And A. Williams, An Overview of Trilinos, Tech. Rep. SAND20032927, Sandia National Laboratories, 2003.

[30] J. E. Hilliard AND J. W. CAHn, An evaluation of procedures in quantitative metallography for volume-fraction analysis, vol. 221, General Electric Research Laboratory, Schenectady, N.Y., 1961.

[31] M. Hintermüller, M. Hinze, And M. H. TBer, An adaptive finiteelement Moreau-Yosida-based solver for a non-smooth Cahn-Hilliard problem, Optim. Methods Softw., 26 (2011), pp. 777-811.

[32] M. Hintermüller, K. Ito, And K. Kunisch, The primal-dual active set strategy as a semismooth Newton method, SIAM J. Optim., 13 (2002), pp. 865-888.

[33] M. Hintermüller And M. Ulbrich, A mesh-independence result for semismooth newton methods, Math. Program., 101 (2004), pp. 151-184.

[34] D. KAY AND R. Welford, A multigrid finite element solver for the Cahn-Hilliard equation, J. Comput. Phys., 212 (2006), pp. 288-304. 
[35] J. Kim, K. Kang, And J. Lowengrub, Conservative multigrid methods for Cahn-Hilliard fluids, J. Comput. Phys., 193 (2004), pp. 511-543.

[36] R. Kornhuber, Monotone multigrid methods for elliptic variational inequalities I, Numer. Math., 69 (1994), pp. 167-184.

[37] P. Lions And B. Mercier, Splitting algorithms for the sum of two nonlinear operators, SIAM J. Numer. Anal., 16 (1979), pp. 964-979.

[38] A. Novick-Cohen, The Cahn-Hilliard equation: Mathematical and modeling perspectives, Adv. Math. Sci. Appl., 8 (1998), pp. 965-985.

[39] Y. Oono And S. PuRI, Study of phase-separation dynamics by use of cell dynamical systems. I. Modeling, Phys. Rev. A, 38 (1988), pp. 434453.

[40] R. L. Pego, Front migration in the nonlinear Cahn-Hilliard equation, Proc. R. Soc. Lond., Ser. A, 422 (1989), pp. 261-278.

[41] J. W. Ruge and K. StüBen, Algebraic multigrid, in Multigrid methods, vol. 3 of Frontiers Appl. Math., SIAM, Philadelphia, PA, 1987, pp. $73-130$.

[42] Y. SAAD, Iterative methods for sparse linear systems, SIAM, Philadelphia, PA, 2003.

[43] Y. SaAd And M. H. Schultz, GMRES: a generalized minimal residual algorithm for solving nonsymmetric linear systems, SIAM J. Sci. Stat. Comput., 7 (1986), pp. 856-869.

[44] G. Strang and G. Fix, An Analysis of the Finite Element Method 2nd Edition, Wellesley-Cambridge, 2nd ed., May 2008.

[45] M. UlbRICH, Semismooth Newton methods for operator equations in function spaces, SIAM J. Optim., 13 (2002), pp. 805-842.

[46] H. A. VAn Der Vorst, BiCGSTAB: A fast and smoothly converging variant of biCG for the solution of nonsymmetric linear systems, SIAM J. Sci. Stat. Comput., 13 (1992), pp. 631-644.

[47] P. Wesseling, An introduction to multigrid methods, Pure and Applied Mathematics (New York), John Wiley \& Sons Ltd., Chichester, 1992. 
[48] X. Wu And Y. Dzenis, Phase-field modeling of the formation of lamellar nanostructures in diblock copolymer thin films under inplanar electric fields, Phys. Rev. E: Stat., Nonlinear, Soft Matter Phys., 77 (2008), p. 031807. 